\title{
Collaborative Assessment and Management of Suicidality in an Inpatient Setting: Results of a Pilot Study
}

\author{
Thomas E. Ellis \\ The Menninger Clinic, Houston, Texas and Baylor College of \\ Medicine-Houston \\ Jon G. Allen \\ The Menninger Clinic, Houston, Texas and Baylor College of \\ Medicine-Houston
}

\author{
Kelly L. Green \\ University of Pennsylvania-Philadelphia
}

David A. Jobes

Catholic University of America-Washington, DC

\author{
Michael R. Nadorff \\ Baylor College of Medicine-Houston
}

\begin{abstract}
Patients hospitalized for psychiatric reasons exhibit significantly elevated risk of suicide, yet the research literature contains very few outcome studies of interventions designed for suicidal inpatients. This pilot study examined the inpatient feasibility and effectiveness of The Collaborative Assessment and Management of Suicidality (CAMS), a structured evidence-based method for risk assessment and treatment planning (Jobes, 2006). The study used an open-trial, case-focused design to assess an inpatient adaptation of CAMS, spread over a period averaging 51 days. The intervention was provided via individual therapy to a convenience sample of 20 patients ( 16 females and four males, average age 36.9) who were hospitalized with recent histories of suicidal ideation and behavior. Results showed statistically and clinically significant reductions in depression, hopelessness, suicide cognitions, and suicidal ideation, as well as improvement on factors considered "drivers" of suicidality. Treatment effect sizes were in the large range (Cohen's $d>.80$ ) across several outcome measures, including suicidal ideation. Although these findings must be considered preliminary due to the lack of a randomized control group, they merit attention from clinicians working with patients at risk for suicide. This study also supports the feasibility of implementing a structured, suicide-specific intervention for at-risk patients in inpatient settings.
\end{abstract}

Keywords: suicide, CAMS, inpatient, psychotherapy, outcome

\begin{abstract}
Although psychiatric illness is known to be associated with significantly elevated rates of suicide (Tanney, 1992), remarkably little research exists describing treatments that effectively reduce risk of future suicidal behavior (Comtois \& Linehan, 2006). This is especially true in the case of inpatient treatment; for example, a review of the literature on treatment of suicidal individuals (Line-
\end{abstract}

Thomas E. Ellis, The Menninger Clinic, Houston, Texas and Department of Psychiatry and Behavioral Sciences, Baylor College of Medicine; Kelly L. Green, Department of Psychiatry, University of Pennsylvania; Jon G. Allen, The Menninger Clinic and Department of Psychiatry and Behavioral Sciences, Baylor College of Medicine; David A. Jobes, Department of Psychology, Catholic University of America, Washington, DC; Michael R. Nadorff, Department of Psychiatry and Behavioral Sciences, Baylor College of Medicine.

This study was made possible through the generous support of The Menninger Clinic Foundation. The authors thank Elizabeth Conaway, LCSW, Patty Daza, PhD, Donna Lamb, LCSW, Mary Oxford, PhD, and Harrell Woodson, PhD, who served as study therapists. The authors thank also thank Steve Herrera, BSMT, for assistance with data management and analysis.

Correspondence concerning this article should be addressed to Thomas E. Ellis, The Menninger Clinic, 12301 Main Street, Houston, TX 77035. E-mail: tellis@menninger.edu han, 2000) listed only two outcome studies of inpatient treatments for suicidal patients. This suggests a serious gap in the literature, given that suicide risk is especially high for hospitalized psychiatric patients (Nordentoft \& Mortensen, 2007). Indeed, it is estimated that between $4 \%$ and $7 \%$ of the 32,000 suicides in the United States each year occur in inpatient psychiatric settings (Wolfersdorf, 2000). Moreover, the weeks following discharge from a psychiatric hospital are associated with greatly elevated risk of suicidal events (Deisenhammer, Huber, Kemmler, Weiss, \& Hinterhuber, 2007; Kan, Ho, Dong, \& Dunn, 2007).

Although a variety of therapies have been shown effective in reducing symptoms of psychiatric disorders, few have been found effective specifically in reducing suicidal behavior (Linehan, 2000; Slee, Spinhoven, Garnefski, \& Arensman, 2008). However, recent years have seen the introduction and testing of new therapies focused on suicide. For example, Linehan and associates tested a therapy designed specifically for suicidal patients with borderline personality disorder (Dialectical Behavior Therapy [DBT]) and found it superior to community treatment by experts (CTBE; Linehan et al., 2006). Treatment effects after 1 year for medical risk (a combination of suicidal behavior and nonsuicidal self-injury) were significantly greater for DBT $(d=.46)$ compared with CTBE $(d=.27)$. DBT also 
produced significant reductions in suicidal ideation $(d=.97)$, reasons for living $(d=.50)$, and depressive symptoms $(d=$ .93), although these changes were not significantly greater than those associated with CTBE. This was a dismantling study, results of which indicated that "the efficacy of DBT cannot reasonably be attributed solely to general factors associated with receiving expert psychotherapy (p. 763)."

Another study, testing Beck's cognitive model of suicide (Wenzel, Brown, \& Beck, 2008), used community therapists to provide a brief, structured cognitive therapy (CBT) designed to develop alternate coping behaviors to suicidality in patients with recent, severe suicide attempts (Brown et al., 2005). The comparison group was treated with enhanced usual care (EUC), which included tracking and referral services. The investigators found a $50 \%$ reduced risk for future suicide attempts in the cognitive therapy condition compared with EUC. Results at 3-months showed a significant advantage to $\mathrm{CBT}$ on the Beck Depression Inventory-II (BDI-II; CBT $d=0.96$; EUC $d=0.70$ ), Beck Hopelessness Scale (BHS; CBT $d=0.77$; EUC $d=0.42$ ), and the Scale for Suicide Ideation (CBT $d=0.15$; EUC $d=0.11$; Brown et al., 2005).

These and other studies have contributed to a growing body of evidence indicating that outpatient therapies targeting psychological vulnerabilities and suicidal behaviors are effective and produce benefits superior to conventional therapies (Ellis, 2006). These therapies view the suicidal coping response as the primary focus of treatment, rather than as a symptom of an illness that, when treated, will eliminate suicide risk as well.

The picture regarding inpatient treatment, however, is less clear. In a review of psychosocial treatments for suicidal patients, Comtois and Linehan (2006) concluded that"... while inpatient treatment is the standard of care, it has never been found efficacious in a clinical trial (p. 166)." In contrast, there is some evidence that specific therapies delivered in inpatient settings may be beneficial. For example, Bohus et al. (2004) tested DBT in an inpatient setting with 31 patients over a period of 3 months and found substantial benefit compared with a usual treatment group. However, the suicide-related behavior of interest in this study was parasuicide (deliberate self-harm without intent to die), so the question of reduction in ideation and behavior associated with a wish to die still awaits study.

A recently developed protocol adapts the cognitive therapy intervention used in the Brown et al. (2005) study to a brief stay inpatient setting (Ghahramanlou-Holloway, Cox, \& Greene, in press). This intervention, Post Admission Cognitive Therapy (PACT), condenses the 10 outpatient sessions of the Brown et al. (2005) protocol to six sessions of 60 to 90 minutes over three consecutive days. Emphases include development of a collaborative therapeutic alliance, construction of a cognitive-behavioral conceptualization, instilling hope, practicing alternate coping skills, and developing explicit relapse prevention plans. This intervention is currently in early stages of feasibility testing.

In addition to therapies specifically tailored to the vulnerabilities of suicidal patients, Jobes (2006) has developed a system that serves both as a means of assessing risk in suicidal patients and as a platform for developing the kind of therapeutic relationship needed for working effectively with patients who often have low motivation for accepting help (Ellis, 2004). This system, Collaborative Assessment and Management of Suicidality, provides a method for therapists to join with the patient in a collaborative endeavor to develop a shared understanding of the suicidal episode, explore the degree of risk for self-harm, and develop a plan for ensuring the patient's safety. Studies to-date have shown promise for this approach, including evidence suggesting that patients resolve suicidality more quickly using this approach compared with conventional interventions (Jobes, Wong, Conrad, Drozd, \& Neal-Walden, 2005).

The emphasis in Collaborative Assessment and Management of Suicidality on collaborative assessment dovetails with current trends in the assessment literature. The Suicide Status Form (SSF) is a focal point in the initial phase of treatment. Sitting side-byside, the patient and therapist assess several key contributors to the suicidal state: psychological pain, stress, agitation, hopelessness, and self-hate. Patients rate pertinent scales and provide narrative responses for these items. Then they explore wishes to live and wishes to die, and the patient is asked to indicate the "one thing" that would help him or her no longer feel suicidal. Together with a review of the patient's history of suicidal behavior, this exploration leads to a plan for intervention.

The CAMS approach exemplifies the spirit of therapeutic assessment that Finn (2007) articulated: the clinician is not merely collecting information but rather is engaging the patient in a collaborative effort to explicate and understand the patient's experience. Thus, the assessment process is intrinsically psychotherapeutic. Moreover, in CAMS, the results of the assessment are immediately transparent to the patient. CAMS also bears the hallmarks of motivational interviewing, which entails nonjudgmental exploration with a "spirit of curiosity" on the part of the therapist (Westra \& Dozois, 2008, p. 44). As in motivational interviewing, CAMS focuses explicitly on ambivalence rather than resistance (Arkowitz \& Miller, 2008). CAMS investigates the extent of ambivalence about life and death while inviting the patient to refrain from acting on the suicidal wish as other options are explored.

As we have described elsewhere (Allen, 2011), this collaborative, inquisitive approach to understanding the suicidal state of mind exemplifies the stance of mentalizing. Thus, consistent with Hilsenroth and Cromer's (2007) review, the CAMS approach uses many forms of early interventions that are likely to build a therapeutic alliance that will facilitate the patient's remaining in treatment and benefiting from it: a collaborative stance, an empathic connection, in-depth exploration, engaging with painful emotions, fostering self-understanding, providing feedback, and collaborating on treatment goals. An abundance of evidence links such empathic engagement and collaboration to treatment outcome (Elliott, Bohart, Watson, \& Greenberg, 2011; Horvath, Del Re, Fluckiger, \& Symonds, 2011; Tryon \& Winograd, 2011).

The purpose of the present study was to assess the feasibility and performance of the CAMS system in the treatment of psychiatric inpatients with current or recent suicidal ideation and behavior. Our goal was to determine whether patients treated with CAMS showed reduced levels of suicidal ideation, and whether they also experienced reduced levels of symptoms associated with suicide risk, particularly depression, hopelessness, and self-hate. The study was conceptualized as a pilot/feasibility study, consistent with Stage Ia of treatment development described by Rounsaville, Carroll, \& Onken (2001). Our purpose was to establish 
feasibility of implementation and to obtain preliminary data regarding changes in suicidal ideation and related variables over the course of treatment.

\section{Method}

\section{Participants}

The initial group consenting to participation consisted of 24 inpatients at The Menninger Clinic, a 90-bed, private psychiatric hospital that specializes in the treatment of patients with multiple, treatment-resistant disorders. Average length of stay is 6 to 8 weeks. Among the initial sample, four patients failed to complete the protocol (defined as at least four, 50-minute individual therapy sessions) due to premature discharge, resulting in a final sample size of 20. Participants were 16 females and 4 males, ranging from 21 to 55 years of age $(M=36.90, S D=11.06)$. They were hospitalized with suicidal ideation as a significant aspect of their reasons for admission. The average number of previous psychiatric hospitalizations was 2.8 (range, 1-8). Sixteen participants had made suicide attempts within 2 weeks before admission (10 by overdose, 3 by cutting/stabbing, 2 by hanging, 1 by Russian Roulette). According to retrospective chart reviews, 11 of the 20 participants $(55 \%)$ had prior histories of suicide attempts; an equal percentage had documented histories of nonsuicidal self-injury. Of the five participants without a recent suicide attempt (i.e., within 2 weeks of admission), all had indications of severe and imminent suicide risk. Of these five, the first participant reported a strong wish to die, had devised a plan, and had obtained the means to carry it out; the second had multiple suicide risk factors and had been prevented by family members from walking in front of a car; the third reported recent suicidal ideation in the context of bipolar disorder, cocaine abuse, and prior attempts by overdose; the fourth had a history of five prior attempts, was stockpiling pills, and had begun writing farewell notes to loved ones; and the fifth had obtained a gun with the intent of killing himself, but was interrupted by a visitor. All were judged at significant risk of suicide by their treatment teams and expressed agreement that suicide was a significant issue in their illness and treatment.

Diagnoses were made via clinical interviews by staff psychiatrists using $D S M-I V$ criteria. Sixteen participants (80\%) were diagnosed with primary mood disorders, including major depression, bipolar I disorder, and bipolar II disorder. The four participants without a mood disorder diagnosis had primary diagnoses of bulimia nervosa, panic disorder, anxiety disorder NOS, and posttraumatic stress disorder. Fourteen participants $(70 \%)$ were diagnosed with Axis II disorders; among these, 12 (60\% of the total sample) were diagnosed either with borderline personality disorder or personality disorder NOS with borderline features. All participants had histories of suboptimal response to prior treatments, including inpatient and outpatient psychotherapy and pharmacotherapy. No suicide attempts or acts of nonsuicidal self-harm occurred during the course of the study.

The four patients who did not complete treatment and thus were excluded from analyses differed from those retained on age, $t(22)=-2.62, p=.02$ and gender, $\chi^{2}(1, N=24)=4.89, p=.03$. These individuals tended to be male ( $75 \%$ of excluded group vs. $20 \%$ of those retained) and younger (mean age $=22.0$ years for excluded group vs. 36.90 years for those retained). They did not differ significantly in depression severity at admission, $t(22)=$ $-0.35, p=.73$.

\section{Study Therapists}

Study therapists were experienced clinical psychologists and clinical social workers prepared to deliver CAMS by reading the CAMS treatment manual (Jobes, 2006) and by attending a full-day training workshop with the developer of CAMS (D.J.). The CAMS team met weekly to review use of the approach and discuss cases. Video recordings of clinicians using CAMS were reviewed by Dr. Jobes and a senior-level doctoral student in clinical psychology using the CAMS Rating Scale (previous research had established their interrater reliability of the adherence approach, Comtois et al., 2011). Adherent therapists thus received an overall "satisfactory" rating using this approach; feedback was provided to providers when there was a need to increase adherence.

Treatment specifics were articulated in a treatment manual describing the Menninger-specific version of CAMS, dubbed "CAMS-M" (Ellis, Daza, \& Allen, in press).

\section{Procedure}

The study used an open-trial, case series design. Although a majority of patients participating in the study had histories of suicide attempts, patients without recent attempts were considered eligible for the study if current suicidal ideation was a significant part of their reason for admission. Inclusion criteria otherwise were deliberately broad; among patients with suicidal ideation, only those who were actively psychotic or under age 18 were excluded. Availability of CAMS was made known to hospital clinicians through an assortment of channels, including professional discipline meetings and in-service training programs. In addition, a member of the research team also serves as coordinator of individual therapy services and was able to monitor availability of study therapists and make matches with appropriate patients. Patients were informed of the study by the therapist during an early session, given a copy of the consent form, and asked to review it and let the therapist know their decision at the next therapy session. Patients were informed that receiving CAMS was not contingent upon their consenting to the study; they would receive CAMS if they wished, and the consent merely allowed the researchers to collect assessment data for the purpose of analysis and publication. The study was approved by the Institutional Review Board of the Baylor College of Medicine.

In addition to CAMS-M, patients participated in a therapeutic milieu and received the standard array of services for Menninger inpatients. Although these vary somewhat depending on patient need and program enrollment, services generally include group psychotherapy, psychoeducational groups (e.g., stress management, sleep hygiene, etc.), family consultation, and discharge planning. Addictions and eating disorder tracks are also available according to patient need.

Psychopharmacological treatment is virtually universal among Menninger patients, who typically are admitted to the hospital already on multiple medications, including antidepressants, anxiolytics, mood stabilizers, and/or sleep medications. There were no exceptions to this rule in this study sample; all were taking psychotherapeutic medications on admission, and all but one were 
receiving multiple medications. All participants received medication changes over the course of hospitalization, which may have included dosage adjustments, discontinuations, and/or starting of new medications. All study participants were taking multiple psychotherapeutic medications at discharge.

The CAMS-M intervention was provided via two 50-minute individual therapy sessions per week. The first session following consent was devoted to completion of the initial SSF (Jobes, 2006). The briefer SSF tracking form (consisting only of ratings on the core "drivers" and probability of suicide) was administered once per week. All other measures were administered biweekly.

\section{Measures}

Depression. The BDI-II (Beck, Steer, \& Brown, 1996), a 21-item self-report inventory of depressive symptomatology, was used to measure depression. It is one of the most widely used research instruments for this purpose and has demonstrated good psychometric properties for use with inpatient populations (Cole, Grossman, Prilliman, \& Hunsaker, 2003). Each item is rated on a Likert scale from 0 to 3 , with higher scores indicating more severe levels of depressive symptoms. Previous studies have reported BDI-II means of 12.75 in a nonclinical student sample (Carmody, 2005) and of 21.02 in an inpatient sample (Steer, Rissmiller, Ranieri, \& Beck, 1994).

Hopelessness. The BHS (Beck \& Steer, 1993a), consists of 20 true-false items pertaining to future outlook. Hopelessness has been shown to mediate the relationship between depression and suicidality (Beck, 1963; Beck, Kovacs, \& Weissman, 1975). Higher scores on the BHS indicate greater hopelessness severity. Previous studies have reported BHS means of 2.92 in a nonclinical student sample (Kelly, Rollings, \& Harmon, 2005) and of 7.78 in an inpatient sample (Steer, Rissmiller, Ranieri, \& Beck, 1994). The BHS has adequate psychometric properties and has been shown to be predictive of eventual suicide in psychiatric inpatients (Beck, Brown, \& Steer, 1989; Beck, Steer, Kovacs, \& Garrison, 1985).

Suicidal ideation. Suicidal thinking was measured using the Beck Scale for Suicide Ideation (BSS), a 19-item self-report measure (Beck \& Steer, 1993b). This is a well-standardized instrument widely used by researchers and clinicians to quantify severity of suicidal ideation (Beck, Brown, \& Steer, 1997). Items are scored on a Likert scale ranging from 0 to 2 , with higher scores indicating more severe suicidal ideation. Previous studies have reported BSS means of 2.82 in a nonclinical student sample (Hirsch \& Conner, 2006) and of 15.63 in an inpatient sample (Steer, Rissmiller, Ranieri, \& Beck, 1993).

Suicidal cognition. Suicide-relevant cognition was assessed using the Suicide Cognitions Scale, a 20-item, self-report measure containing two subscales: Unbearability (of pain and distress) and Unlovability (Rudd, Schmitz, McClenen, Joiner, \& Elkins, 2008). Items are scored on a Likert scale of 0 to 5 , with higher scores indicating greater levels of suicidal cognitions. Rudd et al. (2008) reported mean total scores from a nonclinical student sample of 22.71 and from an inpatient sample of 53.20.

Therapeutic relationship. Quality of the therapeutic relationship was measured with the Working Alliance Inventory, Short Form (WAI-S). The WAI-S is a 12-item form of the WAI, a self-report inventory that measures the quality of the therapeutic relationship from the patient's perspective (Horvath \& Greenberg,
1989). The comparability of the WAI-S to the original was demonstrated by Busseri and Tyler (2003). Inventory items are rated on a Likert scale of 1 (never) to 7 (always), with higher scores indicating a greater perceived alliance. Munder and associates (Munder, Wilmers, Leonhart, Linster, \& Barth, 2010) reported that the WAI-S showed good psychometric properties when used with inpatient and outpatient samples; they reported an overall mean item score of $3.6(S D=0.83)$ for psychiatric inpatients.

Suicide "drivers". The SSF, an integral part of the CAMS method, is administered in a collaborative process with the patient, as described in the CAMS treatment manual (Jobes, 2006). The SSF is a multifaceted instrument; at its core are five items asking for subjective ratings (0-5) of negative states: psychological pain, stress, agitation, hopelessness, and self-hate. In an inpatient sample of suicidal patients, means for each of the items were as follows: pain: 3.82 ; stress: 3.87 ; agitation: 2.90 ; hopelessness: 3.81 ; and self-hate: 3.74 (Conrad et al., 2009). This set of variables has shown good validity and reliability with both suicidal outpatients (Jobes, Jacoby, Cimbolic, \& Hustead, 1997) and inpatients (Conrad et al., 2009). The first three variables (pain, stress, and agitation) are based on Shneidman's cubic model of psychic pain that lies at the heart of his formulation of the suicidal experience (Shneidman, 1993). The SSF also obtains ratings of the patient's sense of hopelessness, based on the work of Aaron Beck (Beck et al., 1989) and self-hate, derived from Baumeister's work conceptualizing suicide as an escape from the pain of self-loathing (Baumeister, 1990).

\section{CAMS Components, Process, and Treatment}

CAMS is a therapeutic framework that modifies how clinicians engage, assess, and treat suicidality (Jobes, 2006). As noted earlier, CAMS is initiated with the collaborative completion of the SSF, which guides assessment, treatment planning, tracking of risk, and the outcome/disposition of care. Portions of the SSF are completed jointly by clinician and patient, as they endeavor systematically to deconstruct the patient's suicidality through quantitative and qualitative assessments and consideration of empirically based risk factors (e.g., suicidal planning, access to means, attempt history, etc.). The clinician and patient then use the totality of this assessment information to collaboratively develop a suicide-specific/ problem-focused treatment plan that is designed to stabilize the patient through a Crisis Response Plan and the targeting and treating of "drivers" of suicidality-those issues that make the patient suicidal. The intervention also endeavors to help a suicidal patient develop existential purpose and meaning - a life worth living (Jobes, Comtois, Brenner, \& Gutierrez, 2011).

CAMS is thus a philosophy of care as well as a series of specific steps that are guided by the SSF tool. It is intended to build a strong clinical alliance and increase patient motivation and engagement in care. Ongoing CAMS care includes routine suicide risk assessment, further crafting of the Crisis Response Plan, and use of problem-focused interventions to target and treat suicidal drivers. In therapy sessions, clinical adherence to CAMS includes the following:

1. Collaborative Assessment-guided by completion of the SSF. 
2. Collaborative Treatment Planning-using the SSF to develop/ evolve the Crisis Response Plan and the targeting and treating of suicidal drivers.

3. Collaborative Deconstruction of Suicidal Drivers-an ongoing exploration of the functional utility of suicidal coping in the patient's life.

4. Collaborative Problem-Focused Interventions-treating identified suicidal drivers within clinical interventions or referral to external resources.

5. Collaborative Development of Existential Purpose and Meaning - working with the patient to develop a life worth living.

To further illustrate the use of CAMS in clinical practice, Table 1 provides brief examples of clinician statements that are emblematic of the five components of CAMS adherence.

All interventions in CAMS are thus designed to either reduce or eliminate the impact of suicidal drivers (i.e., any suicide-specific contributing factor that leads to suicidal ideation and/or behaviors) and cultivate alternatives to suicidality as a coping response. CAMSbased problem-focused interventions for various suicidal drivers may include targeting and treating hopelessness, emotional dysregulation, interpersonal isolation, impulsivity, symptoms of PTSD, or difficulties imagining and planning for the future. CAMS-based care often leads to the engagement and coordination of related auxiliary services (e.g., psychopharmacology, substance abuse treatment, treatment of health care issues, or vocational counseling).

Unlike other evidence-based suicide-relevant treatments, CAMS is not a new psychotherapy, nor is it limited to a particular therapeutic orientation such as cognitive therapy or psychodynamic therapy. Rather, it is an organizational clinical framework - a therapeutic platform-for maintaining a collaborative focus on the elimination of suicidal ideation and behav- ior as a means of coping. CAMS does not prescribe the specific interventions that the clinician must use. Instead, the CAMS clinician is free to use his or her own expertise and experience to select and implement effective clinical interventions.

The evidence-base for CAMS (and the related use of the SSF) has been growing over the past decade. CAMS is now used in multiple settings in the United States (e.g., Ellis, Allen, Woodson, Frueh, \& Jobes, 2009; Jobes, Bryan, \& Neal-Walden, 2009) as well as other countries (Arkov, Rosenbaum, Christiansen, Jønsson, \& Münchow, 2008; Nielsen, Alberdi, \& Rosenbaum, 2011). The psychometric assessment properties of the SSF have been established and replicated (Conrad et al., 2009; Jobes et al., 1997), and there is good support for the qualitative assessment of the SSF as well (Jobes \& Mann, 1999; Jobes et al., 2004). There are now five published correlational studies supporting the feasibility and clinical value of CAMS and the SSF with suicidal outpatients (Arkov et al., 2008; Jobes et al., 1997; Jobes, Kahn-Greene, Greene, \& Goeke-Morey, 2009; Jobes, Wong, Conrad, Drozd, \& Neal-Walden, 2005; Nielsen et al., 2011).

A recent randomized clinical trial of CAMS both replicates and extends previous correlational support of the approach (Comtois et al., 2011). In this study, CAMS led to rapid and sustained reductions in suicidal ideation and overall symptom distress while increasing reasons for living, optimism, and hope in comparison to treatment as usual. Critically, this clinical trial found that the effectiveness of CAMS in follow-up assessments was most pronounced 12 months after the initial engagement in CAMS care, suggesting both effectiveness and sustained therapeutic impact.

Table 1

Examples of Adherent CAMS-Based Interventions

CAMS intervention

Illustrative therapist statement

Collaborative assessment

"It sounds like you are overwhelmed with misery, so much so that suicide seems a like your only option for relief. I would like to understand more about your pain and despair. May I take a seat next to you so we can complete this assessment tool together? Perhaps that way we can get a deep understand of your suicidal pain and suffering."

Collaborative treatment planning

"Now that we understand how and why you are suicidal, I wonder if we can find a way to keep you safe, not only in the hospital, but also when you return home? I suggest we develop a "Crisis Response Plan," to use if you should have a setback. We can also start addressing issues that cause you to want to kill yourself. In other words, can we figure out together how you can stay safe while we are working on what's behind your suicidality?"

Collaborative deconstruction of suicidal drivers

"Isn't it interesting that you only feel suicidal after a fight with your wife, after which you feel alone in the world, which compels you to drink, and then when you're wasted, you feel these intense suicidal urges? Let's try to connect the dots here and figure out how all this works; perhaps there are other ways for you to find some relief."

Collaborative problem-focused interventions

"Okay so if we get you and your wife into couples therapy, we will be addressing your major suicidal driver-your marital distress. Then, if we can get you back into AA and using your sponsor again, perhaps we can reduce the role that alcohol plays in getting you into a suicidal crisis."

Collaborative development of purpose and meaning

"I'm glad to hear that things are better between you and your wife; and it sounds like AA is helping again, too. Coping is really important. Now, I wonder if we can turn to how you can have more purpose and meaning in your life-even beyond your marriage — so that you have a brighter future and a life more worth living? Perhaps it's possible for you make suicide completely irrelevant." 


\section{Results}

Participants completed an average of 11.1 50-minute sessions of individual therapy, with a range of 4 to 24 sessions. Average length of hospital stay was 51.4 days, ranging from 17 to 99 days. Because of a decreasing sample size over each round of repeated measures (due to variations in length of stay and occasional missed assessments), there was not an adequate sample size to support a repeated-measures analytic strategy. Thus, $t$ tests of pre- versus post-treatment scores were calculated using the first and last assessment score available on each measure (i.e., last observation carried forward) for each participant who had at least two observations on the measure. Effect size (Cohen's $d$; Cohen, 1988) was calculated for each comparison. ${ }^{1}$

As expected, there were significant decreases in depression, hopelessness, suicidal ideation, suicidal cognition, and each of the suicide drivers from the SSF from the first to the last assessment time point (see Table 2). All comparisons were categorized as large, following ranges suggested by Cohen (1988): small, $d=.20$ to .50 ; medium, $d=.50$ to .80 ; large, $d>.80$. The three variables with the largest effect sizes (depressive symptoms, psychological pain, and self-hate) were examined using repeated-measures ANCOVA analyses to examine for possible confounding effects of age and gender. Results for depressive symptoms were consistent with $t$ test analyses and these covariates were nonsignificant. Psychological pain and self-hate were reduced to nonsignificance in this model, although age and gender were also nonsignificant in both analyses. Due to the small sample size, these analyses were underpowered, but overall suggest that age and gender were not significant confounding variables.

Consistent with Jacobson et al. (1999), clinical significance of outcome was calculated for pre- and post-BDI-II scores, as this was the outcome variable on which the most normative data exists. Patients were categorized as having achieved clinically significant change if they had a Reliable Change Index (RCI) score greater than 1.96 (Jacobson \& Truax, 1991) and a final assessment BDI-II score in the normal range (0-13; Beck, Steer, \& Brown, 1996). The percentage of patients who moved into the normal range was $73.6 \%$ (14 of 19 for whom data were available). Fifty-two percent of patients (10 of 19) obtained significant RCI scores; all of these patients $(52.6 \%)$ also scored within the normal range on the BDI-II, signifying clinically significant change. No patient met criteria for deterioration. $^{2}$

Scores on the WAI-S indicated that the therapeutic alliance was rated as strong from the start, increasing over time. With a maximum possible item score of 7 , the individual item mean at the first assessment point was $5.54(n=17 ; S D=1.37)$, with a final assessment mean of $6.08(n=16 ; S D=1.12)$, a significant increase, $t(14)=2.51, p=.025$. The effect size of .44 indicates a small-to-moderate magnitude improvement in working alliance over the course of treatment. Due to potential temporal confounds in the measurement of working alliance and outcome, it has been suggested that an "early session" alliance measurement be used in analyses that seek to examine the effect of working alliance on outcome (e.g., Feeley, DeRubeis, \& Gelfand, 1999; Webb et al., 2011). The association between first assessment WAI-S scores and RCI scores was nonsignificant $(r=-.28, p=.27)$, perhaps due to the apparent ceiling effect in the WAI-S scores.

\section{Discussion}

Results of this open trial of CAMS-based individual therapy with suicidal inpatients provide solid support for further implementation and research on this approach in inpatient psychiatric settings. From an implementation standpoint, CAMS was readily adapted to the inpatient setting; and it was successfully disseminated among a subset of clinicians in a setting in which a more conventional approach to suicidal patients had been previously used. The training was successful in bringing therapists to competency criteria, as indicated by external fidelity checks. The intervention also was well received by patients; although 4 of the original 24 patients ended their hospital stays prematurely, no patient withdrew from the protocol per se, and no adverse reactions were experienced. These findings are especially noteworthy in light of the typical histories of these patients as not having responded to multiple prior interventions, including psychotherapy, pharmacotherapy, and prior hospitalizations.

Of course, it must be noted that length of stay at The Menninger Clinic is unusual; very few psychiatric inpatient services anymore are able to keep patients for more than a few days. Further work is needed to determine whether CAMS can be effectively provided over briefer time periods. Jobes, Comtois, Brenner, and Gutierrez (2011) report work currently taking place in Copenhagen testing a 1 to 3 session version of the CAMS framework for working with suicidal adolescent outpatients. In addition, GhahramanlouHolloway et al. are currently examining a 5-session, inpatient version of Beck and associates' protocol for suicidal inpatients (Ghahramanlou-Holloway et al., in press).

From the standpoint of effectiveness, we found statistically and clinically significant reductions in depression, hopelessness, suicidal ideation, suicide-relevant cognitions, and theorized suicide "drivers" such as psychological pain and self-hate, with large effect sizes. It is instructive to compare these treatment effects with other studies (albeit with different clinical populations): Our effect size for suicidal ideation $(d=1.38)$ is comparable with the effect sizes found by Linehan et al. $(d=0.96 ; 2006)$ and larger than that reported by Brown et al. $(d=0.15 ; 2005)$. Our effect size for depressive symptoms $(d=2.28)$ compares favorably with the studies conducted by Linehan et al. $(d=0.93 ; 2006)$, and Brown et al. $(d=0.96 ; 2005)$. Lastly, our effect size for hopelessness $(d=0.92)$, is comparable with the effect size found by Brown et al. $(d=0.77 ; 2005)$. As such, these preliminary results suggest promise for this approach in effectively reducing, not only current distress and suicidality, but perhaps also risk of future suicidal episodes. Such reduced vulnerability is suggested by reductions in variables known to be associated with suicide risk, notably hopelessness and self-hate, as well as participants' predictions of the probability that they would attempt suicide in the future.

Another positive finding, given the centrality of collaboration in the CAMS framework, is the strengthening of the therapeutic

\footnotetext{
${ }^{1}$ Cohen's $d=M_{\text {pre }}-M_{\text {post }} / \mathrm{SD}_{\text {pooled }}$.

${ }^{2}$ With regard to the intent-to-treat sample for whom data were available ( $n=23$; one patient did not complete assessment before discharge), the percentage of patients who moved into the normal distribution was $69.5 \%$ (16/23). Forty-eight percent (11/23) of the group achieved reliable change, all of whom also normalized, resulting in a $48 \%$ rate of clinically significant change in the intent-to-treat sample (11/23).
} 
Table 2

Pre- and Post-Treatment Means and Mean Comparisons

\begin{tabular}{|c|c|c|c|c|c|c|}
\hline & Pre-treatment Mean $(S D)$ & Post-treatment Mean $(S D)$ & $t$ & $d f$ & $p$ & Cohen's $d$ \\
\hline \multicolumn{7}{|l|}{ Beck scales } \\
\hline BDI-II $(n=19)$ & $35.66(11.29)$ & $9.63(11.54)$ & 8.16 & 18 & $<.001$ & 2.28 \\
\hline BHS $(n=15)$ & $10.67(6.42)$ & $5.33(5.16)$ & 3.94 & 14 & .001 & 0.92 \\
\hline $\operatorname{BSS}(n=15)$ & $12.60(9.50)$ & $2.67(4.08)$ & 4.39 & 14 & .001 & 1.38 \\
\hline \multicolumn{7}{|l|}{ SSF drivers } \\
\hline Pain $(n=20)$ & $4.20(0.77)$ & $1.90(1.29)$ & 6.91 & 19 & $<.001$ & 2.17 \\
\hline Stress $(n=20)$ & $4.06(0.98)$ & $2.49(1.17)$ & 5.59 & 19 & $<.001$ & 1.45 \\
\hline Agitation $(n=20)$ & $3.95(1.32)$ & $2.10(1.37)$ & 4.71 & 19 & $<.001$ & 1.38 \\
\hline Hopelessness $(n=20)$ & $3.92(1.32)$ & $1.95(1.36)$ & 5.72 & 19 & $<.001$ & 1.47 \\
\hline Self-hate $(n=20)$ & $3.90(1.33)$ & $1.73(1.03)$ & 6.38 & 19 & $<.001$ & 1.47 \\
\hline Suicide probability $(n=20)$ & $2.95(1.43)$ & $1.50(0.76)$ & 5.45 & 19 & $<.001$ & 1.27 \\
\hline \multicolumn{7}{|l|}{ SCS } \\
\hline Total scale $(n=15)$ & $49.40(21.28)$ & $28.27(10.15)$ & 4.05 & 14 & .001 & 1.27 \\
\hline Unlovability $(n=15)$ & $30.40(14.19)$ & $18.27(6.56)$ & 3.50 & 14 & .004 & 1.10 \\
\hline Unbearability $(n=15)$ & $19.00(8.23)$ & $10.00(4.34)$ & 4.54 & 14 & .001 & 1.37 \\
\hline
\end{tabular}

Note. $\quad$ BDI-II $=$ Beck Depression Inventory-II; BHS = Beck Hopelessness Scale; BSS = Beck Scale for Suicidal Ideation; SCS $=$ Suicide Cognition Scale.

alliance over the course of treatment, even in the context of initially high alliance ratings. This would seem to be evidence that therapists, consistent with the CAMS model, were focusing effectively on partnering with patients around the issues of suicide and safety. Reasons for the absence of the expected association between alliance and outcome are less clear. A "ceiling effect" on alliance scores, together with the small sample size, likely played a role, but the fact that the correlation trended negative suggests a need for a more nuanced explanation. Although a positive association between alliance and outcome is a consistent finding in psychotherapy outcomes research (e.g., Horvath et al., 2011), it is possible that the association operates differently in an inpatient environment. For example, Blais, Jacobo, \& Smith (2010) also found a negative correlation between alliance and outcome in an inpatient sample, using different measures of alliance and outcome. They interpreted the finding as a reflection of higher functioning patients (who tend to give higher alliance ratings) requiring less improvement before discharge relative to lower functioning patients. Further testing of CAMS hypotheses regarding the centrality of therapeutic alliance in working with suicidal inpatients is needed to satisfactorily answer questions related to this issue.

Overall, this study is a meaningful addition to the existing literature in that it adds to a small but growing body of work showing the utility of a therapeutic intervention specifically focusing on the suicidal coping response. This is in contrast to a more conventional view of suicidality as a symptom expected to diminish as the underlying condition is treated. Moreover, this is the first study of which we are aware to examine outcomes of a CAMS-based intervention in a psychiatric inpatient setting, and one of only a handful of studies of any suicide-focused intervention in inpatient settings.

Its merits notwithstanding, this study suffers from limitations consistent with most pilot studies. In particular, without a comparison group, it is not possible to claim that symptom improvement was caused specifically by the CAMS intervention or that these results are any better than would have occurred through treatment as usual or the mere passage of time. The fact that inpatient treatment is intensive and multifaceted, including biolog- ical and psychosocial interventions, as well as respite from the demands of daily life, precludes confident assertions about causes of improvement without randomization to comparison groups. In addition, the small sample size precludes exploration for what specifics mechanisms hypothesized by CAMS might have mediated or moderated improvement relative to vulnerabilities to suicide.

Finally, the study is limited by the lack of follow-up data after participants were discharged from the hospital, so we were not able to examine whether treatment gains were maintained and whether treatment with CAMS was associated with fewer suicidal episodes after discharge. Further research with larger samples, including comparison conditions, is needed to understand more fully the impact of the CAMS approach. Additionally, future study should include follow-up assessments to examine whether gains achieved in treatment with CAMS are maintained over an extended period following treatment termination and lead to real changes in future suicidal behavior.

\section{References}

Allen, J. G. (2011). Mentalizing suicidal states. In K. Michel, \& D. A. Jobes (Eds.), Building a therapeutic alliance with the suicidal patient (pp. 81-91). Washington, DC: American Psychological Association. doi:10.1037/12303-005

Arkov, K., Rosenbaum, B., Christiansen, L., Jønsson, H., \& Münchow, M. (2008). Treatment of suicidal patients: The collaborative assessment and management of suicidality. Ugeskrift for Laeger, 170, 149-153.

Arkowitz, H., \& Miller, W. R. (2008). Learning, applying, and extending motivational interviewing. In H. Arkowitz, H. A. Westra, W. R. Miller, \& S. Rollnick (Eds.), Motivational interviewing in the treatment of psychological problems (pp. 1-25). New York: Guilford Press.

Baumeister, R. F. (1990). Suicide as escape from self. Psychological Review, 97, 90-113. doi:10.1037/0033-295X.97.1.90

Beck, A. T., Brown, G., \& Steer, R. A. (1989). Prediction of eventual suicide in psychiatric inpatients by clinical ratings of hopelessness. Journal of Consulting and Clinical Psychology, 57, 309-310. doi: 10.1037/0022-006X.57.2.309

Beck, A. T., Brown, G. K., \& Steer, R. A. (1997). Psychometric characteristics of the scale for suicide ideation with psychiatric outpatients. 
Behaviour Research and Therapy, 35, 1039-1046. doi:10.1016/S00057967(97)00073-9

Beck, A. T., Kovacs, M., \& Weissman, A. (1975). Hopelessness and suicidal behavior: An overview. Journal of the American Medical Association, 234, 1146-1149. doi:10.1001/jama.234.11.1146

Beck, A. T., Steer, R. A., \& Brown, G. K. (1996). Manual for the Beck Depression Inventory-II. San Antonio, TX: Psychological Corporation.

Beck, A. T., Steer, R. A., Kovacs, M., \& Garrison, B. (1985). Hopelessness and eventual suicide: A !0-year prospective study of patients hospitalized with suicidal ideation. American Journal of Psychiatry, 142, 559563.

Beck, A. T., \& Steer, R. A. (1993a). Manual for the Beck Hopelessness Scale. San Antonio, TX: The Psychological Corporation.

Beck, A. T., \& Steer, R. A. (1993b). Manual for the Beck Scale for Suicide Ideation. San Antonio, TX: The Psychological Corporation.

Beck, A. T. (1963). Thinking and distortion. Archives of General Psychiatry, 4, 561-567. doi:10.1001/archpsyc.1961.01710120031004

Blais, M. A., Jacobo, M. C., \& Smith, S. R. (2010). Exploring therapeutic alliance in brief inpatient psychotherapy: A preliminary study. Clinical Psychology \& Psychotherapy, 17, 386-394. doi:10.1002/cpp.666

Bohus, M., Haaf, B., Simms, T., Limberger, M. F., Schmahl, C., Unckel, C., ... Linehan, M. (2004). Effectiveness of inpatient dialectical behavioral therapy for borderline personality disorder: A controlled trial. Behaviour Research and Therapy, 42, 487-499. doi:10.1016/S00057967(03)00174-8

Brown, G. K., Have, T. T., Henriques, G. R., Xie, S. X., Hollander, J. E., \& Beck, A. T. (2005). Cognitive therapy for the prevention of suicide attempts: A randomized controlled trial. Journal of the American Medical Association, 294, 563-570. doi:10.1001/jama.294.5.563

Busseri, M. A., \& Tyler, J. D. (2003). Interchangeability of the Working Alliance Inventory and the Working Alliance Inventory, short form. Psychological Assessment, 15, 193-197. doi:10.1037/1040-3590 .15 .2 .193

Carmody, D. P. (2005). Psychometric characteristics of the Beck Depression Inventory-II with college students of diverse ethnicity. International Journal of Psychiatry in Clinical Practice, 9, 22-28. doi:10.1080/ 13651500510014800

Cohen, J. (1988). Statistical power analysis for the behavioral sciences (2nd ed.). Hillsdale, NJ: Erlbaum.

Cole, J. C., Grossman, I., Prilliman, C., \& Hunsaker, E. (2003). Multimethod validation of the Beck Depression Inventory-II and GrossmanCole Depression Inventory with an inpatient sample. Psychological Reports, 93, 1115-1129.

Comtois, K. A., Jobes, D. A., O'Connor, S., Atkins, D. C., Janis, K., Chessen, C., ... Yuodelis, F. C. (2011). Collaborative assessment and management of suicidality (CAMS): Feasibility trial for next-day appointment services. Depression and Anxiety, 28, 963-972

Comtois, K. A., \& Linehan, M. M. (2006). Psychosocial treatments of suicidal behaviors: A practice-friendly review. Journal of Clinical Psychology, 62, 161-170. doi:10.1002/jclp.20220

Conrad, A. K., Jacoby, A. M., Jobes, D. A., Lineberry, T. W., Shea, C. E., Ewing, T., ... Kung, S. (2009). A psychometric investigation of the Suicide Status Form II with a psychiatric inpatient sample. Suicide and Life-Threatening Behavior, 39, 307-320. doi:10.1521/suli.2009 .39.3.307

Deisenhammer, E. A., Huber, M., Kemmler, G., Weiss, E. M., \& Hinterhuber, H. (2007). Psychiatric hospitalizations during the last 12 months before suicide. General Hospital Psychiatry, 29, 63-65. doi:10.1016/ j.genhosppsych.2006.09.007

Elliott, R., Bohart, A. C., Watson, J. C., \& Greenberg, L. S. (2011). Empathy. In J. C. Norcross (Ed.), Psychotherapy relationships that work: Evidence-based responsiveness (2nd ed., pp. 132-152). New York: Oxford University Press. doi:10.1093/acprof:oso/ 9780199737208.003.0006
Ellis, T. E., Allen, J. G., Woodson, H., Frueh, B. C., \& Jobes, D. A. (2009). Implementing an evidence-based approach to working with suicidal inpatients. Bulletin of the Menninger Clinic, 73, 339-354. doi:10.1521/ bumc.2009.73.4.339

Ellis, T. E., Daza, P., \& Allen, J. (in press). Collaborative Assessment and Management of Suicidality at Menninger (CAMS-M): An inpatient adaptation and implementation. Bulletin of the Menninger Clinic.

Ellis, T. E. (2004). Collaboration and a self-help orientation in therapy with suicidal clients. Journal of Contemporary Psychotherapy, 34, 41-57. doi:10.1023/B:JOCP.0000010912.99125.6a

Ellis, T. E. (2006). Epilogue: What have we learned about cognition and suicide and what more do we need to know? In T. E. Ellis (Ed.), Cognition and suicide: Theory, research, and therapy (pp. 369-380). Washington, DC: APA Books. doi:10.1037/11377-017

Feeley, M., DeRubeis, R. J., \& Gelfand, L. A. (1999). The temporal relation of adherence and alliance to symptom change in cognitive therapy for depression. Journal of Consulting and Clinical Psychology, 67, 578-582. doi:10.1037/0022-006X.67.4.578

Finn, S. E. (2007). In our client's shoes: Theory and techniques of therapeutic assessment. Mahwah, NJ: Erlbaum.

Ghahramanlou-Holloway, M., Cox, D. W., \& Greene, F. N. (In press). Post-admission cognitive therapy: A brief intervention for psychiatric inpatients admitted after a suicide attempt. Cognitive and Behavioral Practice. doi:10.1016/j.cbpra.2010.11.006

Hilsenroth, M. J., \& Cromer, T. D. (2007). Clinician interventions related to alliance during the initial interview and psychological assessment Psychotherapy: Theory, Research, Practice, Training, 44, 205-218.

Hirsch, J. K., \& Conner, K. R. (2006). Dispositional and explanatory style optimism as potential moderators of the relationship between hopelessness and suicidal ideation. Suicide and Life-Threatening Behavior, 36 661-667. doi:10.1521/suli.2006.36.6.661

Horvath, A. O., Del Re, A. C., Fluckiger, C., \& Symonds, D. (2011). Alliance in individual psychotherapy. In J. C. Norcross (Ed.), Psychotherapy relationships that work: Evidence-based responsiveness (2nd ed., pp. 25-69). New York: Oxford University Press. doi:10.1093/ acprof:oso/9780199737208.003.0002

Horvath, A. O., \& Greenberg, L. S. (1989). Development and validation of the Working Alliance Inventory. Journal of Counseling Psychology, 36, 223-233. doi:10.1037/0022-0167.36.2.223

Jacobson, N. S., Roberts, L. J., Berns, S. B., \& McGlinchey, J. B. (1999). Methods for defining and determining the clinical significance of treatment effects: Description, application, and alternatives. Journal of Consulting and Clinical Psychology, 67, 300-307. doi:10.1037/0022006X.67.3.300

Jacobson, N. S., \& Truax, P. (1991). Clinical significance: A statistical approach to defining meaningful change in psychotherapy research. Journal of Consulting and Clinical Psychology, 59, 12-19. doi:10.1037/ 0022-006X.59.1.12

Jobes, D. A., Bryan, C. J., \& Neal-Walden, T. A. (2009). Conducting suicide research in naturalistic clinical settings. Journal of Clinical Psychology, 65, 382-395. doi:10.1002/jclp.20556

Jobes, D. A., Comtois, K., Brenner, L., \& Gutierrez, P. (2011). Clinical trial feasibility studies of the Collaborative Assessment and Management of Suicidality (CAMS). In R. C. O'Connor, S. Platt, \& J. Gordon (Eds.), International handbook of suicide prevention: Research, policy, and practice (pp. 383-400). West Sussex, UK: Wiley-Blackwell. doi: 10.1002/9781119998556.ch22

Jobes, D. A., Jacoby, A. M., Cimbolic, P., \& Hustead, L. T. (1997). Assessment and treatment of suicidal clients in a university counseling center. Journal of Counseling Psychology, 44(4), 368-377. doi:10.1037/ 0022-0167.44.4.368

Jobes, D. A., Kahn-Greene, E., Greene, J., \& Goeke-Morey, M. (2009). Clinical improvements of suicidal outpatients: Examining suicide status 
form responses as moderators. Archives of Suicide Research, 13, 147159. doi:10.1080/13811110902835080

Jobes, D. A., \& Mann, R. E. (1999). Reasons for living versus reasons for dying: Examining the internal debate of suicide. Suicide and LifeThreatening Behavior, 29, 97-104.

Jobes, D. A., Nelson, K. N., Peterson, E. M., Pentiuc, D., Downing, V., Francini, K., \& Kiernan, A. (2004). Describing suicidality: An investigation of qualitative SSF responses. Suicide and Life-Threatening Behavior, 34, 99-112. doi:10.1521/suli.34.2.99.32788

Jobes, D. A., Wong, S. A., Conrad, A. K., Drozd, J. F., \& Neal-Walden, T. (2005). The collaborative assessment and management of suicidality versus treatment as usual: A retrospective study with suicidal outpatients. Suicide \& Life-threatening Behavior, 35, 483-497. doi:10.1521/ suli.2005.35.5.483

Jobes, D. A. (2006). Managing suicidal risk: A collaborative approach. New York: Guilford Press.

Kan, C. K., Ho, T. P., Dong, J. Y., \& Dunn, E. L. (2007). Risk factors for suicide in the immediate post-discharge period. Social Psychiatry and Psychiatric Epidemiology, 42, 208-214. doi:10.1007/s00127-0060153-0

Kelly, D. B., Rollings, A. L., \& Harmon, J. G. (2005). Chronic selfdestructiveness, hopelessness, and risk-taking in college students. Psychological Reports, 96, 620-624. doi:10.2466/pr0.96.3.620-624

Linehan, M. M., Comtois, K. A., Murray, A. M., Brown, M., Z., Gallop, R. J., Heard, H. L., . . Lindenboim, N. (2006). Two-year randomized controlled trial and follow-up of dialectical behavior therapy vs. therapy by experts for suicidal behaviors and borderline personality disorder. Archives of General Psychiatry, 63, 757-766. doi:10.1001/archpsyc.63.7.757

Linehan, M. M. (2000). Behavioral treatments of suicidal behaviors: Definitional obfuscation and treatment outcomes. In R. W. Maris, S. S. Canetto, J. L. McIntosh, \& M. M. Silverman (Eds.), Review of suicidology (pp. 84-111). New York: Guilford Press.

Munder, T., Wilmers, F., Leonhart, R., Linster, H., \& Barth, J. (2010). Working Alliance Inventory-Short Revised (WAI-SR): Psychometric properties in outpatients and inpatients. Clinical Psychology \& Psychotherapy, 17, 231-239.

Nielsen, A. C., Alberdi, F., \& Rosenbaum, B. (2011). Collaborative assessment and management of suicidality method shows effect. Danish Medical Bulletin, 58, A4300.

Nordentoft, M., \& Mortensen, P. B. (2007). Hospital admission and suicide in schizophrenia. In R. Tatarelli, M. Pompili, \& P. Girardi (Eds.), Suicide in schizophrenia (pp. 61-76). Hauppauge, NY: Nova Biomedical Books.

Rounsaville, B., Carroll, K. M., \& Onken, L. S. (2001). A stage model of behavioral therapies research: Getting started and moving on from stage
I. Clinical Psychology Science and Practice, 8, 133-142. doi:10.1093/ clipsy.8.2.133

Rudd, M. D., Schmitz, B., McClenen, R., Joiner, T., \& Elkins, G. (2008). Development of a measure of suicide-specific hopelessness: The suicide cognitions scale. Unpublished manuscript.

Shneidman, E. S. (1993). Some controversies in suicidology: Toward a mentalistic discipline. Suicide and Life-Threatening Behavior, 23, 292298.

Slee, N., Spinhoven, P., Garnefski, N., \& Arensman, E. (2008). Emotion regulation as mediator of treatment outcome in therapy for deliberate self-harm. Clinical Psychology \& Psychotherapy, 15, 205-216. doi 10.1002/cpp.577

Steer, R. A., Rissmiller, D. J., Ranieri, W. F., \& Beck, A. T. (1993). Dimensions of suicidal ideation in psychiatric inpatients. Behaviour Research and Therapy, 31, 229-236. doi:10.1016/00057967(93)90090-H

Steer, R. A., Rissmiller, D. J., Ranieri, W. F., \& Beck, A. T. (1994). Use of the computer-administered Beck Depression Inventory and Hopelessness Scale with psychiatric inpatients. Computers in Human Behavior, 10, 223-229. doi:10.1016/0747-5632(94)90005-1

Tanney, B. L. (1992). Mental disorders, psychiatric patients, and suicide. In R. W. Maris, A. L. Berman, J. T. Maltsberger, \& R. I. Yufit (Eds.), Assessment and prediction of suicide (pp. 277-320). New York: Guilford Press.

Tryon, G., \& Winograd, G. (2011). Goal consensus and collaboration. In J. C. Norcross (Ed.), Psychotherapy relationships that work: Evidencebased responsiveness (2nd ed., pp. 153-167). New York: Oxford University Press. doi:10.1093/acprof:oso/9780199737208.003.0007

Webb, C. A., DeRubeis, R. J., Amsterdam, J. D., Shelton, R. C., Hollon, S. D., \& Dimidjian, S. (2011). Two aspects of the therapeutic alliance: Differential relations with depressive symptom change. Journal of Consulting and Clinical Psychology, 79, 279-283. doi:10.1037/a0023252

Wenzel, A., Brown, G. K., \& Beck, A. T. (2008). Cognitive therapy for suicidal patients: Scientific and clinical applications. Washington, DC: APA Books.

Westra, H. A., \& Dozois, D. J. (2008). Integrating motivational interviewing into the treatment of anxiety. In H. Arkowitz, H. A. Westra, W. R. Miller, \& S. Rollnick (Eds.), Motivational interviewing in the treatment of psychological problems (pp. 26-56). New York: Guilford Press.

Wolfersdorf, M. (2000). Suicide among psychiatric inpatients. In K. Hawton \& K. V. Heeringen (Eds.), The international handbook of suicide and attempted suicide (pp. 457-466). West Sussex, England: Wiley. doi:10.1002/9780470698976.ch25

Received August 18, 2011

Revision received November 2, 2011

Accepted November 4, 2011 